\title{
Comment on «Steady Transverse MHD Flow of a Dusty Fluid with Parallel Velocity Fields».
}

\author{
R. M. BARRON \\ Department of Mathematics and Statistics, and Director, \\ Fluid Dynamics Research Institute, University of Windsor \\ Windsor, Ontario NgB $3 P_{4}$, Canada
}

(ricevuto il 3 Giugno 1988)

PACS. 47.65. - Magnetohydrodynamies and electrohydrodynamics.

The above-mentioned paper, authored by S. N. Singh, S. S. Ganwar and R. Babu, appeared in $I l$ Nuovo Cimento in $1981\left(^{(1)}\right.$. A large portion of the paper is a verbatim copy of a paper which I published in Tensor, N.S. in $1977\left(^{2}\right)$. Singh $e t$ $a l$. have added a transverse magnetic field which modifies the momentum equation by a term $\frac{1}{2} \rho^{-1} \nabla\left(B^{2}\right)$, but this term does not play any role in the subsequent analysis. From the bottom of p. 420 onwards, Singh et al. have copied my paper word for word. Incidentally, in Theorems 1, 2 and 3, Singh et al. have added the words «infinitely conducting» to my theorems but have neglected to indicate their validity is restricted to transverse flows.

Finally, I wish to express my sincere gratitude to Prof. R. Ricci and B. Bertotti for the actions they have taken to prevent this situation from reoccurring.

(') S. N. SINGH, S. S. GANWAR and R. BABU: Nuovo Cimento B, 65, 419 (1981).

$\left({ }^{2}\right)$ R. M. BARRon: Tensor, N. S., 31, 271 (1977). 